Instrumental Achievements

- X-Ray Analysis

\title{
Crystal Structure of 2-Difluoromethylthio-4,6-bis(isopropylamino)- 1,3,5-triazine 5-Oxide
}

\author{
Motoo ShIro*, Koichi Morita** and Yoshio HaYASE** \\ *Shionogi Research Laboratories, Shionogi \& Co., Ltd., Fukushima, Osaka 553, Japan \\ **A Aburahi Laboratories, Shionogi Research Laboratories, Koka-cho, Shiga 520-34, Japan
}

2-Methylthio-s-triazine herbicides have been thought to be detoxified in plants and soils by degradative removal of the methylthio groups after the formation of sulfoxide and sulfone derivatives by MFO (mixed function oxidase) oxidation. ${ }^{1}$ However, oxidation of 2-difluoromethylthio-4,6-bis(isopropylamino)-1,3,5-triazine by $m$-chloroperbenzoic acid, assumed to be a model for

\section{Table 1 Crystal and experimental data}

Formula: $\mathrm{C}_{10} \mathrm{H}_{17} \mathrm{~N}_{5} \mathrm{OF}_{2} \mathrm{~S}$

Formula weight $=293.34$

Crystal system: monoclinic

Space group: $P 2_{1} / c \quad Z=8$

$a=16.691(3) \AA$

$b=15.778(3)$

$c=11.506(1)$

$V=2903.2(9) \AA^{3}$

$D_{\mathrm{x}}=1.342 \mathrm{~g} / \mathrm{cm}^{3}$

$R=0.047$

No. of reflections used $=2459$

Measurement: Rigaku AFC-5

Program system: private (XPACK SHIONOGI)

Structure determination: MULTAN84

Refinement: block-diagonal
MFO oxidation, gives its $\mathrm{N}$-oxide. ${ }^{2}$ S-Oxidation is difficult owing to the electron-deficiency of $S$ resulting from the introduction of the $F$ atoms. However, the $\mathrm{N}$ atom oxidized remains to be identified. The present work has confirmed the oxidation product to be the 5-N-oxide derivative.

The crystal of the title compound contains two crystallographically independent molecules, referred to as molecules A and B. The atoms of molecule B are represented by the primed numbers of the corresponding ones of molecule $A$. The $H$ atoms were located on a difference electron density map and refined with isotropic temperature factors set equal to $B$ eq values of the bonded atoms. Molecules A and B are linked by<smiles></smiles>

Fig. I Chemical structure.

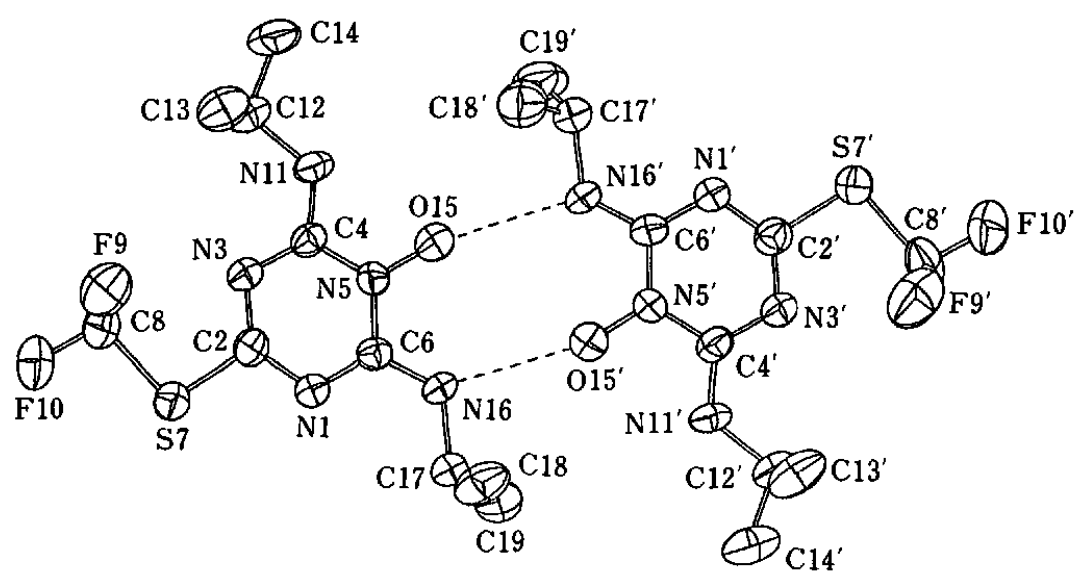

Fig. 2 Perspective view with atom-numbering. 
Table 2 Final coordinates $\left(\times 10^{4}\right)$ of non-H atoms

\begin{tabular}{|c|c|c|c|c|}
\hline & $x$ & $y$ & $z$ & $B_{e q} \times 10^{2} / \AA^{2}$ \\
\hline 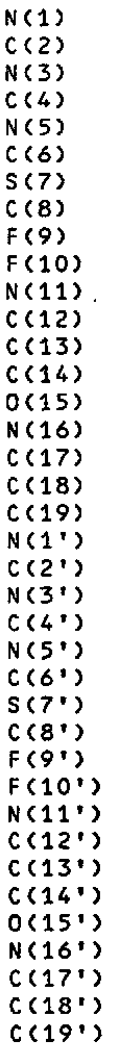 & $\begin{array}{r}9875(1) \\
9336(2) \\
9490(1) \\
10301(1) \\
10898(1) \\
10680(1) \\
8289.0(4) \\
7751(2) \\
7743(1) \\
6951(1) \\
10579(1) \\
10026(2) \\
9572(2) \\
10587(2) \\
11692(1) \\
11297(1) \\
11137(2) \\
11251(3) \\
11723(2) \\
14618(1) \\
15132(2) \\
15013(1) \\
14277(1) \\
13725(1) \\
13900(2) \\
16080.5(4) \\
16613(2) \\
16253(2) \\
17339(2) \\
14041(1) \\
14552(2) \\
14569(3) \\
14169(3) \\
13028(1) \\
13314(1) \\
13408(2) \\
12746(2) \\
13329(3)\end{array}$ & $\begin{array}{c}295(1) \\
585(2) \\
973(1) \\
1071(2) \\
771(1) \\
386(1) \\
423.6(6) 6 \\
867(2) \\
1717(1) \\
628(2) \\
1460(1) \\
1823(2) \\
2605(2) \\
2010(3) \\
842(1) \\
107(1) \\
-293(2) \\
372(3) \\
-1047(2) \\
1788(1) \\
1604(2) \\
1066(1) \\
673(1) \\
794(1) \\
1372(2) \\
2162.9(6) 10 \\
1653(4) \\
2069(2) \\
2074(3) \\
127(1) \\
-60(2) \\
701(3) \\
-844(2) \\
358(2) \\
1475(2) \\
2094(2) \\
2772(2) \\
1614(3)\end{array}$ & $\begin{array}{r}7307(2) \\
6297(2) \\
5367(2) \\
5473(2) \\
6445(2) \\
7365(2) \\
62917(6) \\
4818(3) \\
4885(2) \\
4616(2) \\
4636(2) \\
3500(2) \\
3746(3) \\
2679(3) \\
6476(2) \\
8286(2) \\
9362(2) \\
10354(3) \\
9753(3) \\
8913(2) \\
9992(2) \\
10817(2) \\
10500(2) \\
9391(2) \\
8604(2) \\
0313.5(6) \\
11816(3) \\
12593(2) \\
12132(2) \\
11218(2) \\
12478(2) \\
13292(3) \\
12872(3) \\
9098(2) \\
7565(2) \\
6633(3) \\
6495(4) \\
5475(3)\end{array}$ & $\begin{array}{l}452(6) \\
467(6) \\
436(6) \\
431(6) \\
450(6) \\
427(6) \\
628(2) \\
691(9) \\
985(8) \\
1023(8) \\
514(6) \\
586(8) \\
786(11) \\
838(13) \\
695(6) \\
512(6) \\
615(9) \\
902(14) \\
838(13) \\
489(6) \\
523(7) \\
525(6) \\
494(7) \\
537(6) \\
489(7) \\
677(2) \\
1070(17) \\
1257(10) \\
1500(14) \\
608(7) \\
643(9) \\
925(13) \\
914(15) \\
927(9) \\
623(7) \\
654(9) \\
876(13) \\
934(14)\end{array}$ \\
\hline
\end{tabular}

$B_{\mathrm{eq}}=(4 / 3) \sum_{i j} \sum_{i j} a_{i} a_{j}$

intermolecular hydrogen bonds, $\mathrm{N}(16) \cdots \mathrm{O}\left(15^{\prime}\right)=2.798$ (4) $\AA$ and $N(16) \cdots O(15)=2.820(4) \AA$ (broken lines in Fig. 2), to form a bimolecular unit adopting an approximate $C_{2}$ point symmetry. The corresponding bond dis tances and angles of the molecules agree within experimental error, excluding those relevant to the difluoromethylthio groups which exhibit large thermal motions especially in molecule $B$.

\section{References}

1. See works cited in ref. 2.
Table 3 Bond distances $(\AA)$ and angles $\left({ }^{\circ}\right)$

\begin{tabular}{|c|c|c|c|c|}
\hline \multicolumn{4}{|c|}{ Molecule A } & $\frac{B}{1.324(4)}$ \\
\hline $\begin{array}{l}N(1) \\
N(1) \\
C(2) \\
C(2) \\
N(3) \\
C(4) \\
C(4) \\
N(5) \\
N(5) \\
C(6) \\
S(7) \\
C(8) \\
C(8) \\
N(11 \\
C(12 \\
C(12 \\
N(16 \\
C(17 \\
C(17\end{array}$ & $\begin{array}{l}-C \\
-C \\
-N \\
-S \\
-C \\
-N \\
-N \\
-C \\
-C \\
-N \\
-C \\
-F \\
-F \\
=C \\
\Rightarrow-C \\
\Rightarrow-C \\
\Rightarrow-C \\
\Rightarrow-C\end{array}$ & $\begin{array}{l}(2) \\
(6) \\
(3) \\
(7) \\
(4) \\
5) \\
11) \\
(6) \\
15) \\
(16) \\
(8) \\
(9) \\
10) \\
12) \\
13) \\
(14) \\
(17) \\
18) \\
19)\end{array}$ & $\begin{array}{l}1.331(4) \\
1.332(3) \\
1.321(4) \\
1.764(3) \\
1.333(4) \\
1.352(4) \\
1.332(4) \\
1.358(3) \\
1.321(3) \\
1.324(3) \\
1.816(4) \\
1.344(4) \\
1.342(5) \\
1.482(4) \\
1.516(5) \\
1.538(6) \\
1.480(4) \\
1.522(6) \\
1.523(5)\end{array}$ & $\begin{array}{l}1.324(4) \\
1.323(4) \\
1.330(4) \\
1.757(3) \\
1.331(3) \\
1.356(3) \\
1.329(3) \\
1.374(4) \\
1.310(4) \\
1.320(5) \\
1.883(6) \\
1.378(7) \\
1.339(8) \\
1.486(4) \\
1.518(6) \\
1.520(6) \\
1.493(5) \\
1.514(6) \\
1.505(6)\end{array}$ \\
\hline $\begin{array}{l}C(2) \\
N(1) \\
N(1) \\
N(3) \\
C(2) \\
N(3) \\
N(3) \\
N(5) \\
C(4) \\
C(4) \\
C(6) \\
N(1) \\
N(1) \\
N(5) \\
C(2) \\
S(7) \\
S(7) \\
F(9) \\
C(4) \\
N(11) \\
N(11) \\
C(13) \\
C(6) \\
N(16) \\
N(16) \\
C(18)\end{array}$ & $\begin{array}{l}-N(1) \\
-C(2) \\
-C(2) \\
-C(2) \\
-N(3) \\
-C(4) \\
-C(4) \\
-C(4) \\
-N(5) \\
-N(5) \\
-N(5) \\
-C(6) \\
-C(6) \\
-C(6) \\
-S(7) \\
-C(8) \\
-C(8) \\
-C(8) \\
-N(11) \\
-C(12) \\
-C(12) \\
-C(12) \\
-N(16) \\
-C(17) \\
-C(17) \\
-C(17)\end{array}$ & $\begin{array}{l}-C(6) \\
-N(3) \\
-S(7) \\
-S(7) \\
-C(4) \\
-N(5) \\
-N(11) \\
-N(11) \\
-C(6) \\
-O(15) \\
-O(15) \\
-N(5) \\
-N(16) \\
-N(16) \\
-C(8) \\
-F(9) \\
-F(10) \\
-F(10) \\
-C(12) \\
-C(13) \\
-C(14) \\
-C(14) \\
-C(17) \\
-C(18) \\
-C(19) \\
-C(19)\end{array}$ & $\begin{array}{r}115.2(2) \\
128.9(3) \\
112.0(2) \\
119.1(2) \\
114.0(3) \\
121.6(3) \\
122.8(3) \\
115.6(3) \\
120.2(2) \\
119.3(2) \\
120.5(2) \\
120.0(2) \\
123.1(2) \\
116.9(2) \\
99.9(2) \\
110.0(2) \\
104.8(2) \\
105.3(3) \\
123.8(3) \\
111.6(3) \\
106.3(3) \\
112.5(3) \\
121.8(2) \\
108.6(3) \\
109.0(3) \\
112.6(3)\end{array}$ & $\begin{array}{r}115.9(3) \\
128.4(3) \\
112.9(2) \\
118.7(2) \\
114.6(2) \\
121.1(2) \\
123.1(2) \\
115.7(2) \\
119.9(2) \\
118.8(2) \\
121.3(3) \\
120.0(3) \\
124.1(3) \\
115.9(3) \\
98.0(2) \\
102.3(4) \\
100.9(4) \\
96.7(5) \\
123.0(2) \\
110.5(3) \\
106.2(3) \\
113.3(3) \\
121.9(3) \\
108.3(3) \\
107.8(3) \\
112.8(3)\end{array}$ \\
\hline
\end{tabular}

2. K. Morita, K. Ide and Y. Hayase, Agric. Biol. Chem. [Tokyo], 53, 251 (1989).
(Received July 12,1989 ) (Accepted July 31, 1989) 